\title{
Demonstration of Controlling the Spatiotemporal Dynamics of Optical Near-Field Excitation Transfer in Y-Junction Structure Consisting of Randomly Distributed Quantum Dots
}

\author{
Wataru Nomura, ${ }^{1}$ Makoto Naruse, ${ }^{2}$ Masashi Aono, ${ }^{3,4}$ Song-Ju Kim, ${ }^{5}$ Tadashi Kawazoe, \\ Takashi Yatsui, ${ }^{1}$ and Motoichi Ohtsu ${ }^{1}$ \\ ${ }^{1}$ School of Engineering, The Nanophotonics Research Center, The University of Tokyo, 2-11-16 Yayoi, Bunkyo-ku, Tokyo 113-8656, Japan \\ ${ }^{2}$ Photonic Network Research Institute, National Institute of Information and Communications Technology, 4-2-1 Nukui-kita, \\ Koganei, Tokyo 184-8795, Japan \\ ${ }^{3}$ Earth-Life Science Institute, Tokyo Institute of Technology, 2-12-1 Ookayama, Meguro-ku, Tokyo 152-8550, Japan \\ ${ }^{4}$ PRESTO, Japan Science and Technology Agency, 4-1-8 Honcho, Kawaguchi-shi, Saitama 332-0012, Japan \\ ${ }^{5}$ WPI Center for Materials Nanoarchitectonics (MANA), National Institute for Materials Science, 1-1 Namiki, \\ Tsukuba, Ibaraki 305-0044, Japan \\ Correspondence should be addressed to Wataru Nomura; nomura@nanophotonics.t.u-tokyo.ac.jp
}

Received 10 March 2014; Accepted 24 March 2014; Published 27 April 2014

Academic Editor: Chennupati Jagadish

Copyright (C) 2014 Wataru Nomura et al. This is an open access article distributed under the Creative Commons Attribution License, which permits unrestricted use, distribution, and reproduction in any medium, provided the original work is properly cited.

\begin{abstract}
Solution searching devices that operate on the basis of controlling the spatiotemporal dynamics of excitation transfer via dressed photon interactions between quantum dots have been proposed. Long-range excitation transfer based on dressed photon interactions between randomly distributed quantum dots is considered to be effective in realizing such devices. Here, we successfully controlled the spatiotemporal dynamics of excitation transfer using a Y-junction structure consisting of randomly dispersed CdSe/ZnS core-shell quantum dots. This Y-junction structure has two "output ends" and one "tap end." By exciting one output end with control light, we observed increased excitation transfer to the other output end via a state-filling effect. Conversely, we observed reduced excitation transfer to the output ends by irradiating the tap end with control light, due to excitation of defect levels in the tap end. These results show the possibility of controlling the optical excitation transfer dynamics between multiple quantum dots.
\end{abstract}

\section{Introduction}

Light excitation in quantum dots (QDs) generates dressed photons, which are light fields localized in the vicinity of the QDs, giving rise to dressed photon interactions with other nearby matter, as well as excitation energy transfer via these interactions [1]. In particular, various optical functional devices, such as logic gates called nanophotonic devices [24], light-harvesting devices [5], and optical signal transmitting systems [6, 7], have been realized using QDs formed of $\mathrm{CuCl}, \mathrm{ZnO}$, InAs, CdSe, and so forth, based on optical nearfield excitation transfer between QDs. Nanophotonic devices have been shown to function as logic gates, such as AND, NOT, and XOR logic gates [2-4]. These devices consist of two or three closely spaced QDs having different energy levels, and by inputting a light beam serving as a power supply and another light beam serving as a control signal, excitation energy transfer between the QDs is controlled so that the light emitted from one of the QDs serves as the output.

On the other hand, novel solution searching and decision making devices using a QD array provided with multiple output QDs have recently been proposed [8-10]. In these devices, by inputting control signals to the output QDs based on certain rules, the probability of the optical excitation being transferred uniformly to each QD is controlled to obtain a solution. In these operations, it is necessary to control the spatiotemporal dynamics of the optical excitation transfer between spatially distributed QDs. The features of these 
devices are that they operate at high speed with low energy consumption since they are driven by excitation transfer based on dressed photon interactions [11]. The proposed solution searching device finds a solution to a nondeterministic polynomial (NP) time complete problem, the satisfiability problem (SAT), much faster than the WalkSAT algorithm, which is one of the fastest stochastic local search algorithms $[9,12]$. The proposed decision making device exhibits high efficiency and adaptability in solving decision making problems [10].

However, although it would be ideal to utilize the models discussed in [8-10], it would be technically challenging to directly implement their architecture. One reason for this is that, in order to implement and operate this device, it is essential to fabricate a QD array in which QDs of strictly selected dimensions and number must be precisely located with nanometer-order positioning precision, as reported in [8], which is technologically demanding from the viewpoint of fabrication as the first step. In addition, another reason is the technical challenge of having to guide the control light to multiple output QDs and separately observe the output signals coming from them.

In contrast to this approach, we distributed an extremely large number of identical-size QDs randomly, and by using a structure in which an output QD is provided at the terminal end, we experimentally demonstrated the possibility of optical excitation energy transfer over distances of micrometer order via repeated dressed photon interactions between the QDs [6]. Using such a randomly distributed QD structure overcomes the fabrication difficulties mentioned above, and because of the possibility of scaling up the technique to micrometer order, it is expected that it will also be possible to overcome the technical challenges related to control and observation.

In the work reported in this paper, using a structure in which multiple QDs are randomly distributed, we experimentally investigated control of the spatiotemporal dynamics of optical excitation transfer based on dressed photon interactions between the QDs, which is a requirement for the realization of solution searching and decision making devices. Section 2 describes fundamental principles. Section 3 describes the fabricated device, experimental setup, energy transfer measurement, and characterizations. Section 4 concludes the paper.

\section{Principle}

First, we will explain optical excitation transfer due to dressed photon interactions and suppression of this process. As shown in Figure 1, a small QD (QD1) and a large QD (QD2) are placed in close proximity. A first excited state E11 in QD1 is resonant with a second excited state E22 in QD2 due to their size ratio of $1: 1.43$ [13]. When QD1 is excited by excitation light $P_{\text {in } 1}$, a dressed photon is generated in the vicinity of QD1. The excitation energy in QD1 is transferred to E22 by dressed photon interactions and then relaxes to E21, which is the first excited state in QD2, via intersubband relaxation. Thus, the excitation energy is transferred unidirectionally (Figure 1(a)).
This is observed as emitted light $P_{\text {out2 }}$ from QD2. However, if E21, which is the level to which the excitation relaxes, has already been excited by control light $P_{\text {in2 }}$, the excitation energy transferred to E22 cannot relax to E21, but it is transferred back to E11, and nutation occurs between E11 and E22 (Figure 1(b)). This is observed as emitted light $P_{\text {out1 }}$ from QD1, or when there is a QD in the vicinity, this leads to an increase in the probability of excitation transfer to that QD. Based on this principle, assuming that QD1, having multiple QD2's in the vicinity, is excited, by irradiating one QD2 with $P_{\text {in2 }}$, the probability of excitation transfer to the other QD2's is changed; in other words, it is possible to control the spatiotemporal dynamics.

Next, we will explain optical excitation transfer based on dressed photon interactions in a randomly distributed QD structure. In this structure, as shown in Figure 1(c), identicalsize QDs (QD1) are randomly distributed in close proximity to each other, and QD2's are placed at one location to serve as an output. The input light $P_{\text {in } 1}$ incident on the QD1's at one end undergoes repeated dressed photon interactions between the QD1's and eventually arrives at the QD2's, where it is output as emitted light $P_{\text {out2 }}$ from E21. Because the optical excitation is transferred with high probability by QDs having strong dressed photon interactions, optical excitation transfer with high energy efficiency is possible regardless of the QD1 distribution, and it has been experimentally verified that it is possible to transfer the excitation over a distance of more than $10 \mu \mathrm{m}[6]$.

The experimental model used in this paper is shown in Figure 1(d). The green color in the figure represents a Y-junction QD1 structure consisting of a group of QD1's randomly distributed in close proximity to each other, in the form of strips with a width of $1 \mu \mathrm{m}$ and a length of $7.5 \mu \mathrm{m}$ extending in a radiating pattern in three directions from the center $\mathrm{O}$. The ends of the $\mathrm{Y}$-junction are labeled A, B, and C. QD2 structures serving as output ports, where QD2's are grouped together in squares with a side length of $3 \mu \mathrm{m}$, are connected at $\mathrm{A}$ and $\mathrm{B}$ so as to overlap with the QD1 structure. From A and B, called "output ends", we obtain output signals that are observed as radiation. In addition, $\mathrm{A}$ and $\mathrm{B}$ also serve as terminals for inputting control light. On the other hand, end C, which we call a "tap end," is not connected to any QD2's. The detailed experimental conditions used in this model are omitted. Figure 1(e) shows an energy band diagram in which the section including points A-O-B is illustrated one-dimensionally. When excitation light $P_{\text {inl }}$, serving as an input signal, is incident on the center point $\mathrm{O}$ in the QD1 structure, optical excitation is generated in QD1 and is transferred through the QD1 structure by undergoing repeated energy transfer due to dressed photon interactions between the QD1's. Since the optical excitation transferred to QD2 reaches E21 via intersubband relaxation, no reverse flow of the transfer occurs, and therefore the optical excitation is transferred to the ends, $\mathrm{A}$ and $\mathrm{B}$, where the QD2 structures are provided. At this time, if end B is irradiated with control light $P_{\text {in2 }}$ that excites QD2, QD2 is excited, and the excitation light transferred from QD1 does not relax to E21, and thus excitation transfer is not observed. Therefore, a reverse flow of the optical excitation occurs at 


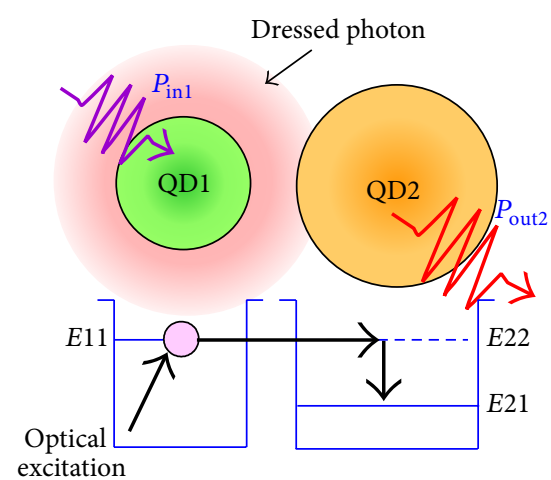

(a)

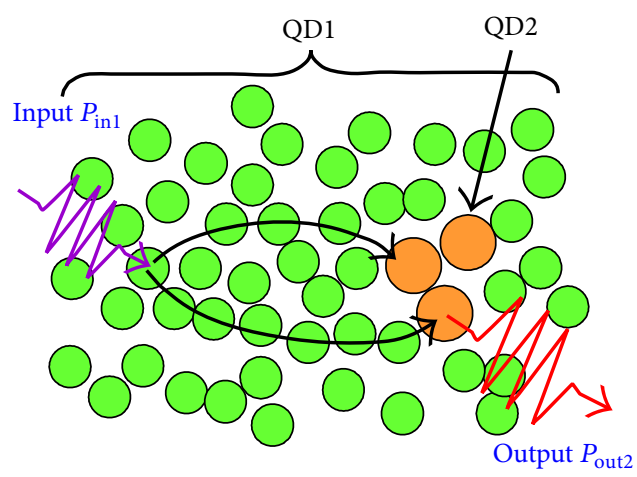

(c)

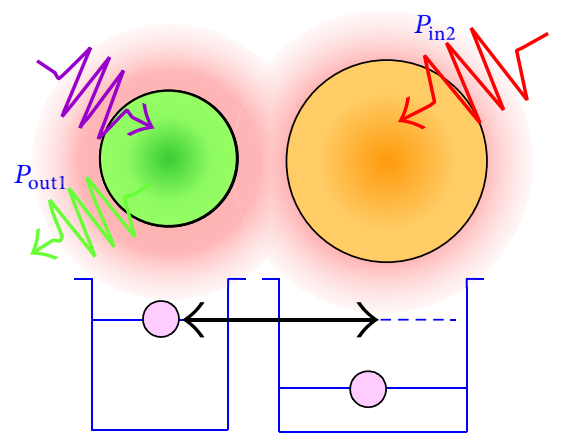

(b)

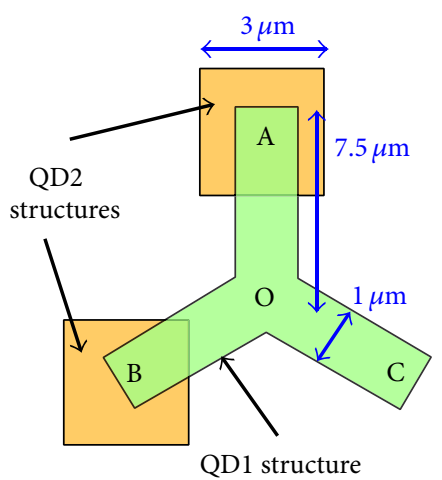

(d)

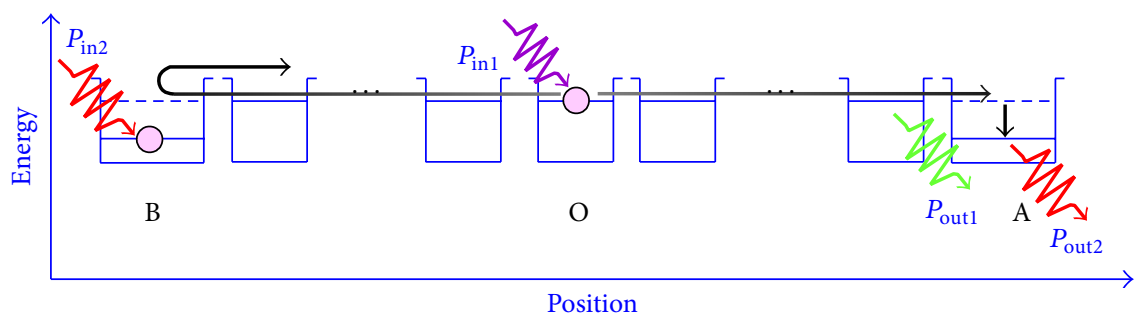

(e)

FIgURE 1: (a), (b) Schematic illustration of optical excitation transfer from QD1 to QD2 via dressed photon interactions, where (a) shows the case where energy transfer occurs from QD1 to QD2 and (b) shows the case where control light is incident on QD2, thus suppressing energy transfer. (c) Schematic diagram of long-range optical excitation transfer system. Green and yellow circles represent QD1's and QD2's, respectively. (d) Schematic diagram of experimental model. Green and yellow regions represent groups of QD1 and QD2 structures consisting of randomly distributed QD1's and QD2's, respectively. (e) Energy diagram schematically showing the experimental model from point B to point A.

end $\mathrm{B}$, and the level of excitation transfer to $\mathrm{A}$, which is the other output end, increases. At locations where the excitation transfer is increased, the emitted light $P_{\text {out1 }}$ and $P_{\text {out2 }}$ from QD1 and QD2 both increase, and, therefore, we can confirm that the excitation transfer is controlled by evaluating the increase in $P_{\text {out1 }}$ at end $\mathrm{A}$ when end $\mathrm{B}$ is irradiated with control light $P_{\text {in2 }}$. Note that even if end $\mathrm{C}$ is irradiated with control light $P_{\mathrm{in} 2}$, a change like that occurring at points $\mathrm{A}$ and $\mathrm{B}$ is not expected to occur since the excitation state of the QDs does not change.

\section{Experiments}

3.1. Device. In our experiments, we used commercially available CdSe/ZnS core-shell QDs (manufactured by Quantum Design, Inc.). As QD1 and QD2 described in the previous section, we selected QDs with core-diameters of $d_{1}=2.5 \mathrm{~nm}$ and $d_{2}=3.2 \mathrm{~nm}$, respectively. Their first excited states, estimated from absorption spectra, were E11 $=2.36 \pm 0.08 \mathrm{eV}$ and E21 $=$ $2.11 \pm 0.06 \mathrm{eV}$, respectively. As described above, E11 is resonant with E22, which is the second excited state of QD2 [14]. 


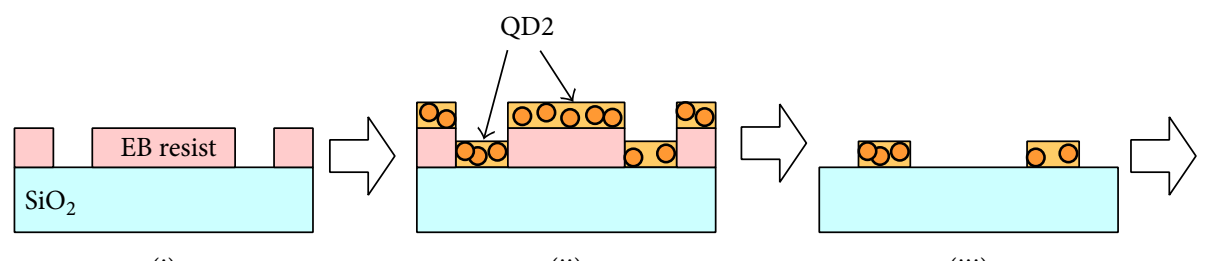

(i)

(ii)

(iii)

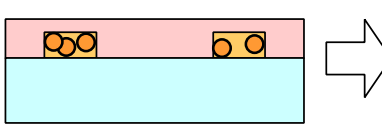

(iv)

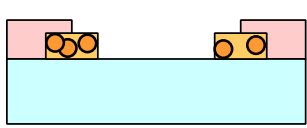

(v)

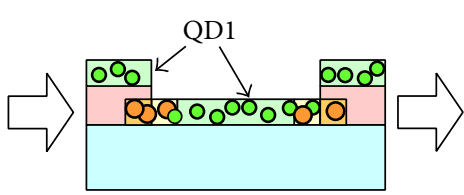

(vi)

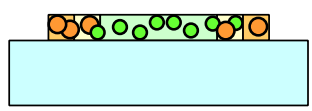

(vii)

(a)

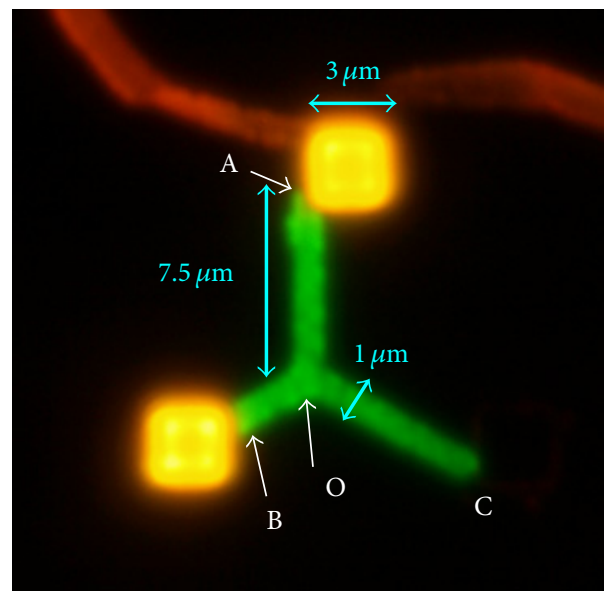

(b)

FIgURE 2: (a) Cross-sectional illustrations of sample fabrication process. (i) E-beam resist is applied to $\mathrm{SiO}_{2}$ substrate, and regions where QD2 structures are to be formed are drawn by e-beam lithography. (ii) QD2 solution is dropped onto substrate and allowed to dry. (iii) E-beam resist is removed to obtain QD2 structures. (iv) E-beam resist is applied again. (v) Region where QD1 structure is to be formed is drawn by e-beam lithography. (vi) QD1 solution is dropped onto resist and allowed to dry. (vii) E-beam resist is removed to obtain the sample. (b) Fluorescence microscope image of sample excited by mercury vapor lamp.

The outside of the shell was modified with a carboxyl group. The solution was dropped onto a substrate and allowed to dry, affording a group of randomly distributed QDs located in close proximity with a center-to-center distance of about 10 to $20 \mathrm{~nm}$.

We fabricated samples using the procedure shown in Figure 2(a). First, by using e-beam lithography on a silica substrate, in e-beam resist (ZEP-520A, Zeon Corp.), we drew locations at which QD2's were to be deposited (Figure 2(a)(i)). Next, the QD2 solution was dropped onto the substrate and allowed to dry (Figure 2(a)(ii)). Then, after removing the resist, we obtained the QD2 structure on the substrate (Figure 2(a)(iii)). Next, e-beam resist was applied on this structure (Figure 2(a)(iv)), and using e-beam lithography, we drew Y-junction locations where QD1's were to be deposited by aligning the positions so as to contact the QD2 structure (Figure 2(a)(v)). After developing the resist, we dropped the QD1 solution onto the substrate (Figure 2(a)(vi)). Finally, the resist was removed to obtain the Y-junction QD1 structure (Figure 2(a)(vii)). Figure 2(b) shows a fluorescence microscope image obtained when the fabricated sample was excited with a mercury-vapor lamp. The QD1 structure and the QD2 structure were observed as green and yellow colors, respectively. Because points $\mathrm{A}$ and $\mathrm{B}$ were set at positions where the QD1 and QD2 structures were initially in contact, in practice the distances $\mathrm{OA}$ and $\mathrm{OB}$ of the fabricated samples were about $6.8 \mu \mathrm{m}$ and $3.3 \mu \mathrm{m}$, respectively. Point $\mathrm{C}$, which did not possess the QD2 structure, was set at a position $7 \mu \mathrm{m}$ away from O. 


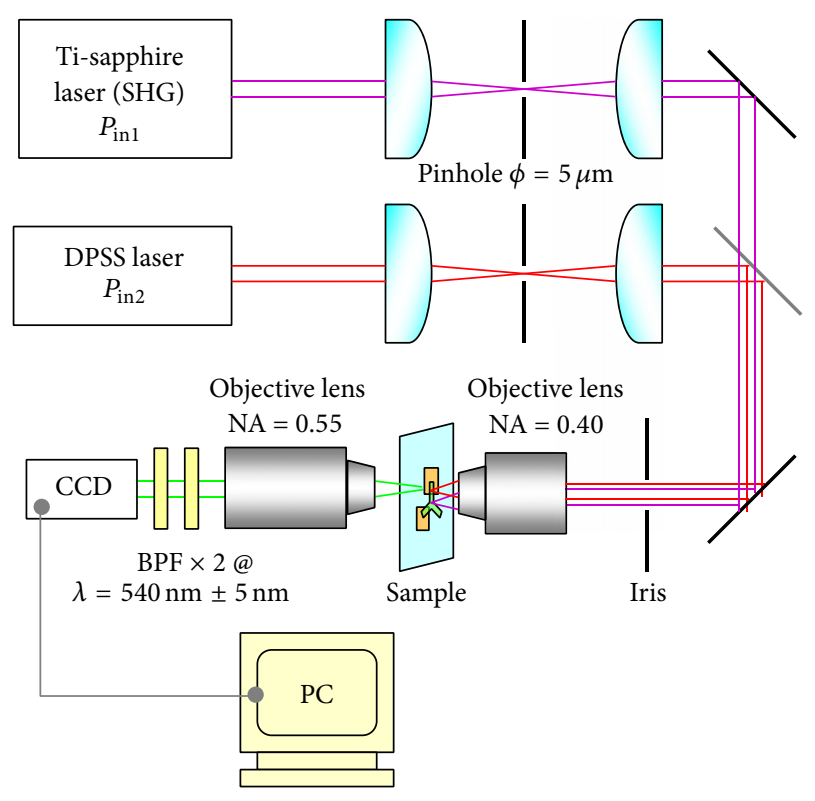

FIGURE 3: Schematic diagram of experimental setup for measurement.

3.2. Experimental Setup. The experimental setup used for performing measurements is shown in Figure 3. For the excitation light $P_{\text {in1 }}$, we used the 2 nd harmonic wave of a mode-locked $\mathrm{Ti}$ : sapphire laser (Mira 900, Coherent, Inc.) with a photon energy $h \nu_{1}=3.44 \mathrm{eV}$, a pulse width of $2 \mathrm{ps}$, and a repetition frequency of $80 \mathrm{MHz}$, and for the control light $P_{\text {in2 }}$, we used a CW diode-pumped solid state (DPSS) laser with a photon energy $h \nu_{2}=2.11 \mathrm{eV}$. The incident powers of $P_{\text {in } 1}$ and $P_{\text {in } 2}$ were $5 \mu \mathrm{W}$ and $10 \mu \mathrm{W}$, respectively, and the beam spot diameters were both $5 \mu \mathrm{m}$. The beams from the two lasers were shaped by passing them through pinholes with $5 \mu \mathrm{m}$ diameter apertures; then, using a half-mirror, the beams irradiated the sample from the back surface of the substrate via an objective lens with a numerical aperture $\mathrm{NA}=0.4$. As described in the previous section, because it is possible to observe the change in excitation transfer at the output end as a variation in the emitted light $P_{\text {outl }}$ from QD1, we evaluated this in order to make it easier to separate it from the control light. The light emitted from QD1 was observed using an electron multiplying CCD camera (Hamamatsu ImagEM C9100-13H, Hamamatsu Photonics K.K.) after passing through an objective lens with $\mathrm{NA}=0.55$ and two bandpass filters with transmission wavelengths of $540 \pm 5 \mathrm{~nm}$, which is close to the wavelength of $P_{\text {out } 1}$. The acquired images were $512 \times 512$ pixel, 16-bit grayscale images, and the resolution was $0.37 \mu \mathrm{m} /$ pixel.

As an example of the measurement results, Figure 4(a) shows a CCD image acquired when $\mathrm{O}$ and $\mathrm{A}$ were irradiated with excitation light $P_{\text {in } 1}$ and control light $P_{\text {in2 } 2}$, respectively. The green and yellow broken lines show the QD1 and QD2 structures, respectively. Point $\mathrm{O}$ was irradiated with $P_{\text {in } 1}$, and we observed that the light emitted from the QD1 structure spread out in three directions due to transfer of the excitation. A cross-sectional brightness profile taken along the light-blue dotted line in this figure is shown by the red solid line in
Figure 4(b). The black solid line shows the case where point $\mathrm{O}$ was irradiated with only $P_{\text {in } 1}$. The red solid line is higher in a portion of the QD1 structure serving as the transfer path $\mathrm{OB}$ and the point $\mathrm{B}$ having the QD2 structure, confirming the tendency for the optical excitation directed towards point $\mathrm{B}$ to be increased due to the influence of $P_{\text {in2 }}$ incident on point A.

3.3. Energy Transfer in Y-Junction Structure. To evaluate the intensity of the emitted light, we used the brightness values acquired with the CCD camera, where the total brightness of $3 \times 3$ pixels was defined as emission intensity, $I$. While irradiating point $\mathrm{O}$ with $P_{\text {inl }}$, we measured $I_{i j}$, where $i$ indicates the output end $\mathrm{A}$ or $\mathrm{B}$, and $j$ indicates where the control-light $P_{\text {in2 }}$ is irradiated, that is, to the input terminal $\mathrm{A}, \mathrm{B}$, or C. Also, when no control light is irradiated, we represent this by $j=0$. Concerning the characteristics of the device under study, described in Section 3.1, there are four representative categories:

(a) Reference: without any $P_{\text {in2 }}(j=0)$;

(b) Case 1: emission end and control-light input terminal are the same point $(i=j)$;

(c) Case 2: emission end and control-light input terminal are opposite points $((i, j)=(\mathrm{A}, \mathrm{B})$ or $(\mathrm{B}, \mathrm{A}))$;

(d) Case 3: control-light input terminal is point $\mathrm{C}(j=\mathrm{C})$.

The evaluation was performed using the relative intensity change, $\Delta I_{i j}$, defined as $\Delta I_{i j}=\left(I_{i j}-I_{i 0}\right) / I_{i 0}$. At this time, if $\Delta I_{i j}$ is positive in Case 2, excitation transfer from one end at which the control light is input to the other end will be increased, and control of the excitation transfer will be observed.

Figure $4(\mathrm{c})$ shows a plot of $\Delta I_{i j}$ obtained in Cases 1,2 , and 3. First, we evaluated Case 1 (i.e., $i=j$ ), as shown by the blue bars in Figure 4(c); they took positive values $\Delta I_{\mathrm{AA}}=3.9 \times 10^{-2}$ and $\Delta I_{\mathrm{BB}}=3.7 \times 10^{-2}$, confirming that the emitted light was stronger. This is the effect of controlling the excitation transfer, and regarding energy transfer in the regions where QD1 and QD2 coexist, we obtained results showing that it is possible to control the excitation transfer also among multiple QDs. This indicates that an AND-gate operation was manifested with multiple randomly distributed QDs, as reported in [3].

Next, we evaluated Case 2 (i.e., $i \neq j=\mathrm{A}, \mathrm{B}$ ), as shown by the red bars in Figure 4(c); they also took positive values $\Delta I_{\mathrm{AB}}=7.3 \times 10^{-2}$ and $\Delta I_{\mathrm{BA}}=3.1 \times 10^{-2}$, confirming that the emitted light was stronger. $\Delta I_{\mathrm{AB}}$ was higher than $\Delta I_{\mathrm{BA}}$ because the wider overlapped area of QD1 and QD2 structures at point $B$ provided more variation of excitation transfer than that at point $\mathrm{A}$. This difference can be reduced by preparing finer structures and will not disturb the proper operation of solution exploring algorithms. From these results, we confirmed that the excitation transfer was suppressed by the incident control light. These results show that it is possible to control the optical excitation transfer dynamics in a randomly distributed QD structure. Since the relative change $\Delta I$ was reduced by losses involved in the optical excitation transfer, we expect that larger values will 


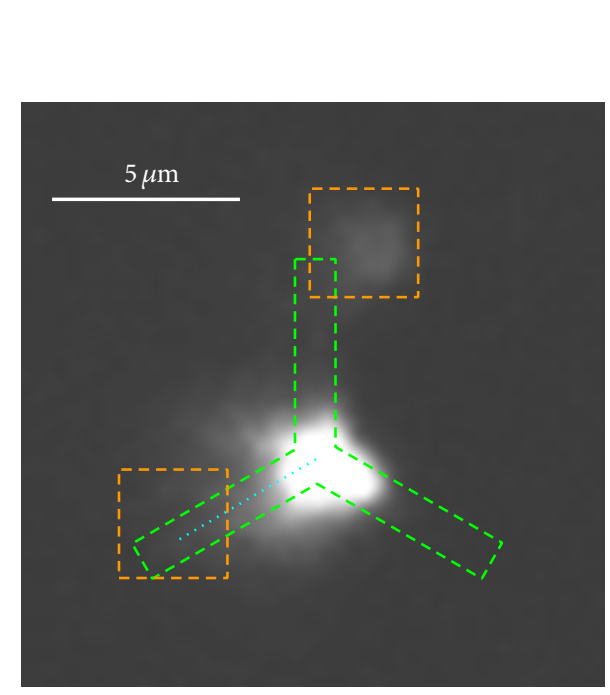

(a)

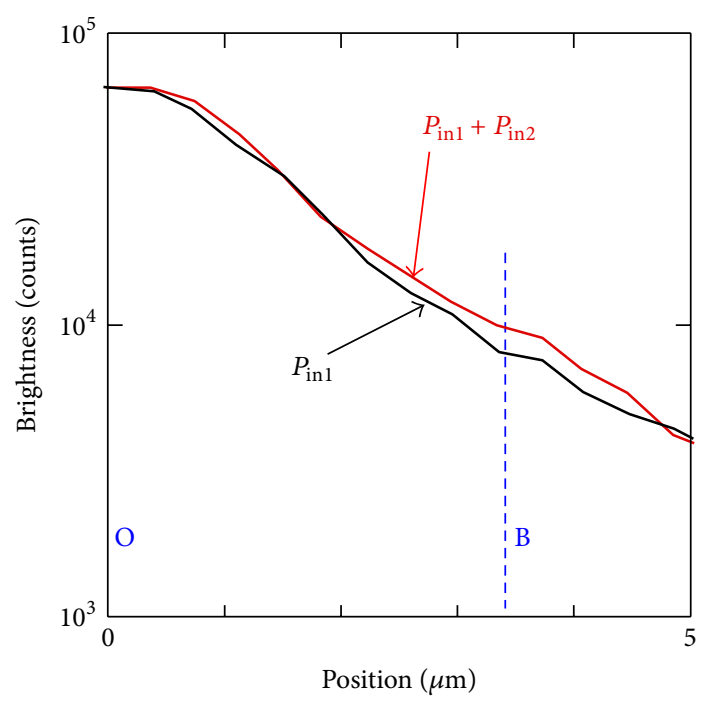

(b)

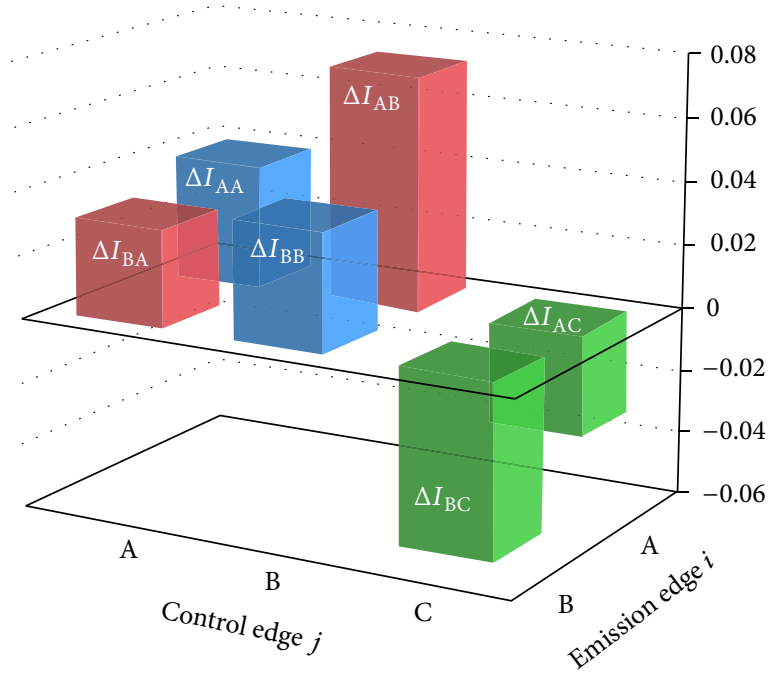

(c)

Figure 4: (a) Image of light emission from E11 in sample, acquired with CCD camera. Points $\mathrm{O}$ and A are irradiated with light $P_{\text {in1 }}$ and $P_{\text {in2 }}$, respectively. Green and yellow broken lines represent the QD1 and QD2 structures, respectively. (b) Cross-sectional profile of emission intensity from E11, acquired with CCD. The red solid line is the cross-sectional profile taken along the light-blue dotted line in (a). The black solid line is the cross-sectional profile taken along the same location, when point $\mathrm{O}$ was irradiated with $P_{\text {inl }}$ only. The $0 \mu \mathrm{m}$ base point and the blue broken line indicate point $\mathrm{O}$ and point B, respectively. (c) Graph of relative change $\Delta I_{i j}$ in emission intensity from E11. Blue, red, and green bars represent Cases 1, 2, and 3, respectively.

be possible by designing a structure having shorter distances $\mathrm{OA}$ and $\mathrm{OB}$.

3.4. Discussion on Negative Intensity Change. Moreover, we evaluated Case 3 (i.e. $j=C$ ), as shown by the green bars in Figure 4(c); they took negative values $\Delta I_{\mathrm{AC}}=-3.2 \times 10^{-2}$ and $\Delta I_{\mathrm{BC}}=-5.5 \times 10^{-2}$. Ideally, QD1 should be transparent to $P_{\mathrm{in} 2}$; however, in our experiments, defect levels with energies lower than E11 in QD1 were excited by $P_{\text {in2 }}[15,16]$. We consider that this excitation prevented the excitation in E11 from slow and nonradiative relaxation via the defect levels.
To verify this, we performed photoluminescence measurements on the QD1 structure. In this experiment, we used samples having only the QD1 structure, fabricated with the same process as that used to fabricate the measurement samples, and we performed measurements with an experimental setup in which the bandpass filters and the CCD camera in the setup shown in Figure 3 were replaced with a spectrometer. The incident powers of $P_{\text {in } 1}$ and $P_{\text {in2 } 2}$ were $5 \mu \mathrm{W}$ and $770 \mu \mathrm{W}$, respectively, and the beams irradiated the sample in spot diameters of $10 \mu \mathrm{m}$. The emission intensities were integrated in the range $2.25<h v<2.29 \mathrm{eV}$, corresponding to the peak wavelength of $P_{\text {out1 }}$, and were normalized to the 


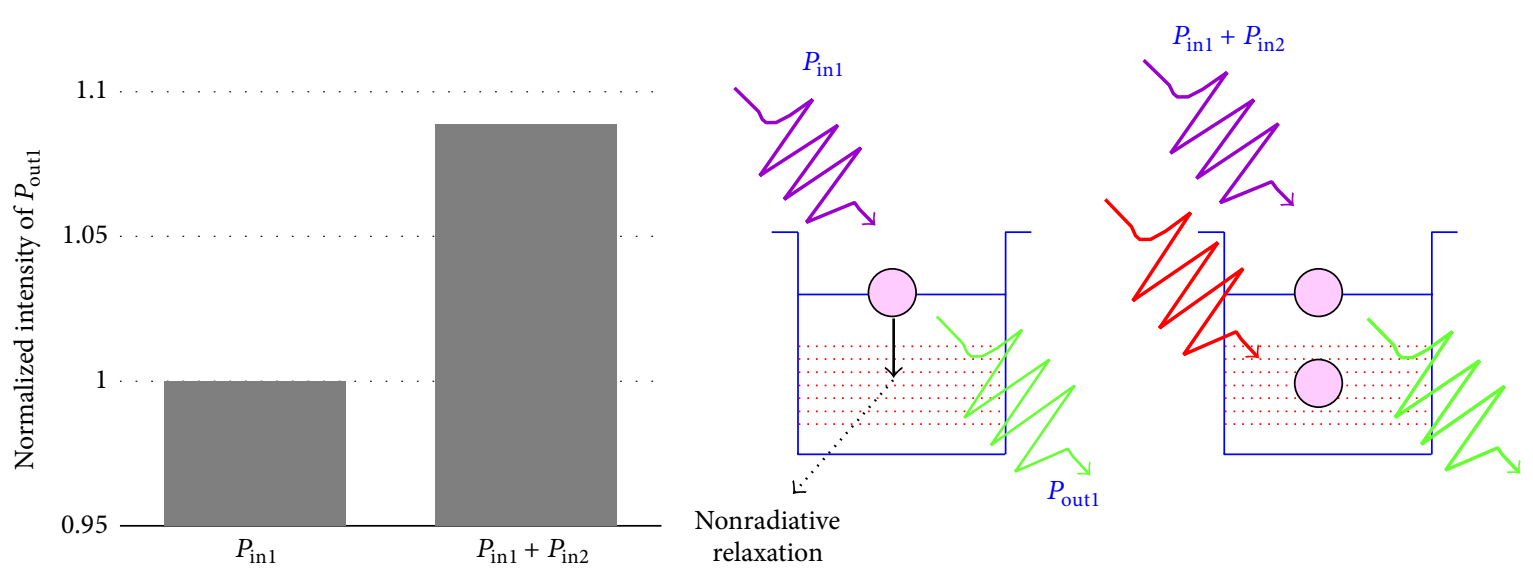

(a)

(b)

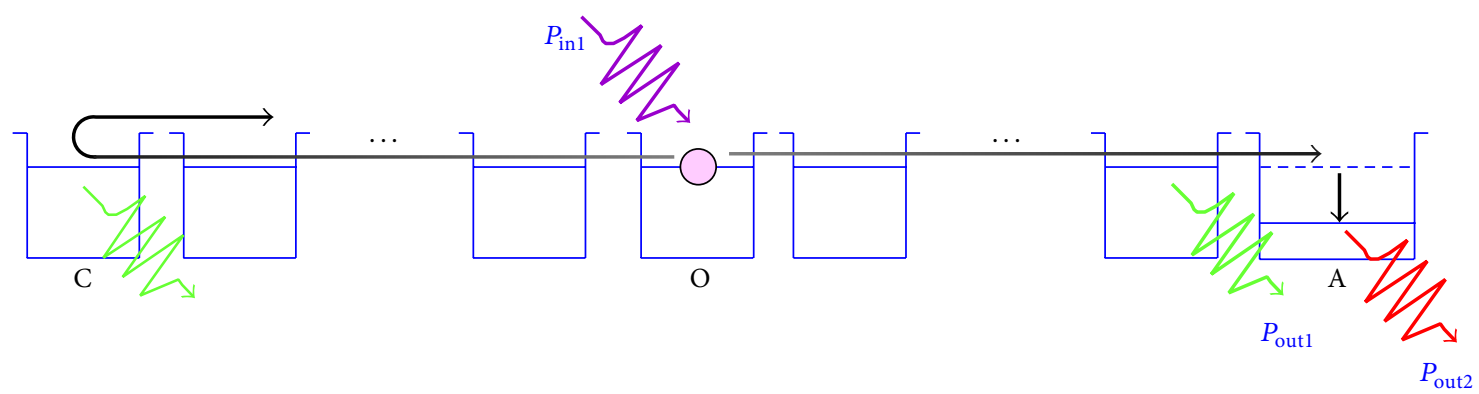

(c)

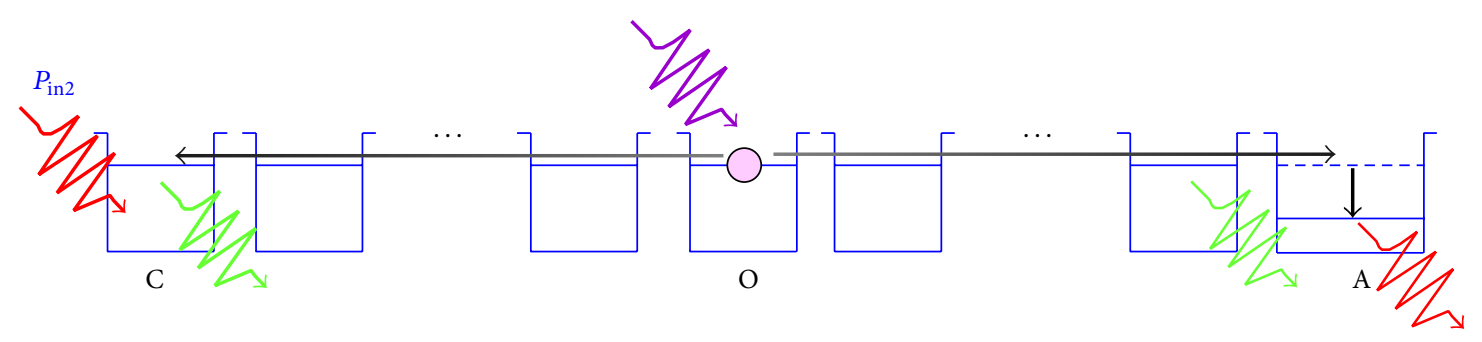

(d)

FIgURE 5: (a) Normalized intensity of emitted light $P_{\text {outl }}$ from E11, showing cases where the QD1 structure was irradiated with only $P_{\text {inl }}$ and with both $P_{\text {in1 }}$ and $P_{\text {in2. }}$. (b) Schematic image of emission from QD1 with only $P_{\text {in1 }}$ or with both $P_{\text {in1 }}$ and $P_{\text {in2. }}$. (c), (d) Energy diagram schematically showing the experimental sample from point C to point A: (c) without $P_{\text {in2 }}$, (d) point C irradiated with $P_{\text {in2 }}$.

emission intensity when only $P_{\text {in } 1}$ was radiated and were compared. As a result, the emission intensity for the case where $P_{\text {in } 1}$ and $P_{\text {in2 }}$ were simultaneously radiated was 1.09 , showing that the emitted light was increased by radiating $P_{\text {in2 }}$ (Figure 5(a)). This was the result of defect levels in QD1 being excited by $P_{\text {in2 } 2}$, suppressing the nonradiative relaxation process from E11 via defect levels, which increased the emitted light (Figure 5(b)).

From this result, the negative $\Delta I$ values in Case 3 can be explained as follows. Without $P_{\text {in2 }}$, the excitation transferred to end $\mathrm{C}$ reflects and enhances $P_{\text {out1 }}$ at ends $\mathrm{A}$ and $\mathrm{B}$ (Figure 5(c)). By irradiating end $\mathrm{C}$ with $P_{\text {in2 } 2}, P_{\text {out } 1}$ at $\mathrm{C}$ increases due to excitation of defect levels in QD1, and the reflection decreases (Figure 5(d)). As a result, $P_{\text {out1 }}$ at ends $\mathrm{A}$ and $\mathrm{B}$ decreases by irradiation of $\mathrm{C}$ with $P_{\text {in2 }}$. This differs from the principle of excitation transfer suppression described in Section 2 and Figure 1(e) but indicates the possibility of another method of controlling the spatiotemporal dynamics of excitation transfer. Also, this increased light emission due to the fact that excitation of defect levels can also occur in QD1 at points $\mathrm{A}$ and B; however, the energy transfer suppression effect due to excitation of E21 should be larger than this because absorption of $P_{\text {in2 }}\left(h v_{2}=2.11 \mathrm{eV}\right)$ in QD2 is 24 times higher than that in QD1. Thus, the effect of excitation of defect levels at ends A and B is negligible.

\section{Summary}

In summary, to show the possibility of controlling the spatiotemporal dynamics of optical excitation transfer based on dressed photon interactions between multiple randomly distributed quantum dots (QDs), we conducted experiments 
to control excitation transfer using randomly distributed QD structures. Using CdSe/ZnS core-shell QDs, we fabricated a Y-junction structure composed of randomly distributed QDs (QD1 structure). Optical excitation incident at the center of this structure was transferred to a QD2 structure serving as an output end located 3-7 $\mu \mathrm{m}$ away, and by irradiating a QD2 structure at another end with control light $P_{\text {in2 }}$, we observed a maximum increase of $7.3 \times 10^{-2}$. Thus, we have shown, for the first time, that it is possible to control the spatiotemporal dynamics of optical excitation transfer in a randomly distributed QD structure. Our findings will lead to simplified implementation and driving of solution searching and decision making devices based on the optical excitation transfer dynamics between QDs and will contribute to their practical realization. On the other hand, when control light $P_{\text {in2 }}$ was incident on the output end that did not have a QD2 structure, we found that the amount of excitation energy transferred to the output end was reduced by a maximum of $5.5 \times 10^{-2}$. This was thought to be because the light emission from QD1 was increased due to excitation of defect levels in QD1, and the amount of excitation energy transferred to the end provided with the QD2 structure serving as an output was reduced. This effect acted in a direction causing a reduction in excitation transfer, opposite to the increased transfer level due to excitation of the QD2 structure, indicating the possibility of a different kind of control of the spatiotemporal dynamics. This result is expected to lead to the development of novel computing device architectures in the future.

\section{Conflict of Interests}

The authors declare that there is no conflict of interests regarding the publication of this paper.

\section{Acknowledgment}

A part of this work was supported by the "Development of Next-generation High-performance Technology for Photovoltaic Power Generation System" Program, NEDO, Japan.

\section{References}

[1] M. Ohtsu, T. Kawazoe, T. Yatsui, and M. Naruse, "Nanophotonics: application of dressed photons to novel photonic devices and systems," IEEE Journal on Selected Topics in Quantum Electronics, vol. 14, no. 6, pp. 1404-1417, 2008.

[2] T. Kawazoe, K. Kobayashi, S. Sangu, and M. Ohtsu, "Demonstration of a nanophotonic switching operation by optical nearfield energy transfer," Applied Physics Letters, vol. 82, no. 18, pp. 2957-2959, 2003.

[3] T. Kawazoe, M. Ohtsu, S. Aso et al., "Two-dimensional array of room-temperature nanophotonic logic gates using InAs quantum dots in mesa structures," Applied Physics B: Lasers and Optics, vol. 103, no. 3, pp. 537-546, 2011.

[4] A. Karimkhani and M. K. Moravvej-Farshi, "Design of threeinput nanophotonic and gates," Journal of the Optical Society of America B: Optical Physics, vol. 26, no. 5, pp. 1084-1090, 2009.

[5] T. Kawazoe, K. Kobayashi, and M. Ohtsu, "Optical nanofountain: a biomimetic device that concentrates optical energy in a nanometric region," Applied Physics Letters, vol. 86, no. 10, Article ID 103102, pp. 1-3, 2005.

[6] W. Nomura, T. Yatsui, T. Kawazoe, M. Naruse, and M. Ohtsu, "Structural dependency of optical excitation transfer via optical near-field interactions between semiconductor quantum dots," Applied Physics B: Lasers and Optics, vol. 100, pp. 181-187, 2010.

[7] P. Holmström, L. Thylén, and A. Bratkovsky, "Composite metal/quantum-dot nanoparticle-array waveguides with compensated loss," Applied Physics Letters, vol. 97, Article ID 073110, 2010.

[8] M. Naruse, M. Aono, S.-J. Kim et al., "Spatiotemporal dynamics in optical energy transfer on the nanoscale and its application to constraint satisfaction problems," Physical Review B, vol. 86, Article ID 125407, 2012.

[9] M. Aono, M. Naruse, S.-J. Kim et al., "Amoeba-inspired nanoarchitectonic computing: solving intractable computational problems using nanoscale photoexcitation transfer dynamics," Langmuir, vol. 29, no. 254, pp. 7557-7564, 2013.

[10] S.-J. Kim, M. Naruse, M. Aono, M. Ohtsu, and M. Hara, "Decision maker based on nanoscale photo-excitation transfer," Scientific Reports, vol. 3, Article ID 2370, 2013.

[11] M. Naruse, H. Hori, K. Kobayashi, P. Holmström, L. Thylén, and M. Ohtsu, "Lower bound of energy dissipation in optical excitation transfer via optical near-field interactions," Optics Express, vol. 18, no. 23, pp. A544-A553, 2010.

[12] U. Schöning, "A probabilistic algorithm for k-SAT and constraint satisfaction problems," in Proceedings of the 40th IEEE Symposium on Foundations of Computer Science, pp. 410-414, 1999.

[13] N. Sakakura and Y. Masumoto, "Persistent spectral-holeburning spectroscopy of $\mathrm{CuCl}$ quantum cubes," Physical Review B-Condensed Matter and Materials Physics, vol. 56, no. 7, pp. 4051-4055, 1997.

[14] C. Trallero-Giner, A. Debernardi, M. Cardona, E. MenéndezProupín, and A. I. Ekimov, "Optical vibrons in CdSe dots and dispersion relation of the bulk material," Physical Review BCondensed Matter and Materials Physics, vol. 57, no. 8, pp. 46644669, 1998.

[15] M. Y. Valakh, Y. G. Sadfyev, N. O. Korsunska et al., "Deeplevel defects in CdSe/ZnSe QDs and giant anti-stokes photoluminescence," Semiconductor Physics, Quantum Electronics and Optoelectronics, vol. 5, no. 3, pp. 254-257, 2002.

[16] M. Danek, K. F. Jensen, C. B. Murray, and M. G. Bawendi, "Synthesis of luminescent thin-film CdSe/ZnSe quantum dot composites using CdSe quantum dots passivated with an overlayer of ZnSe," Chemistry of Materials, vol. 8, no. 1, pp. 173$180,1996$. 

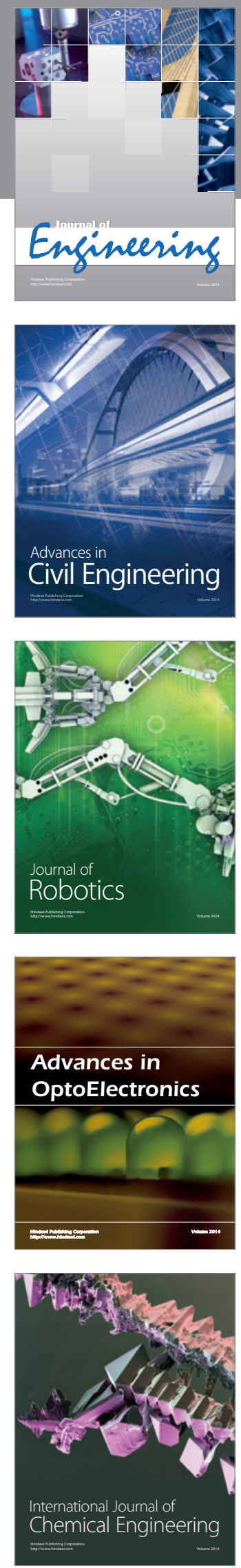

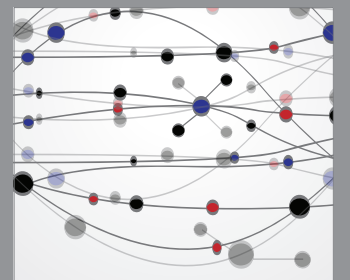

The Scientific World Journal
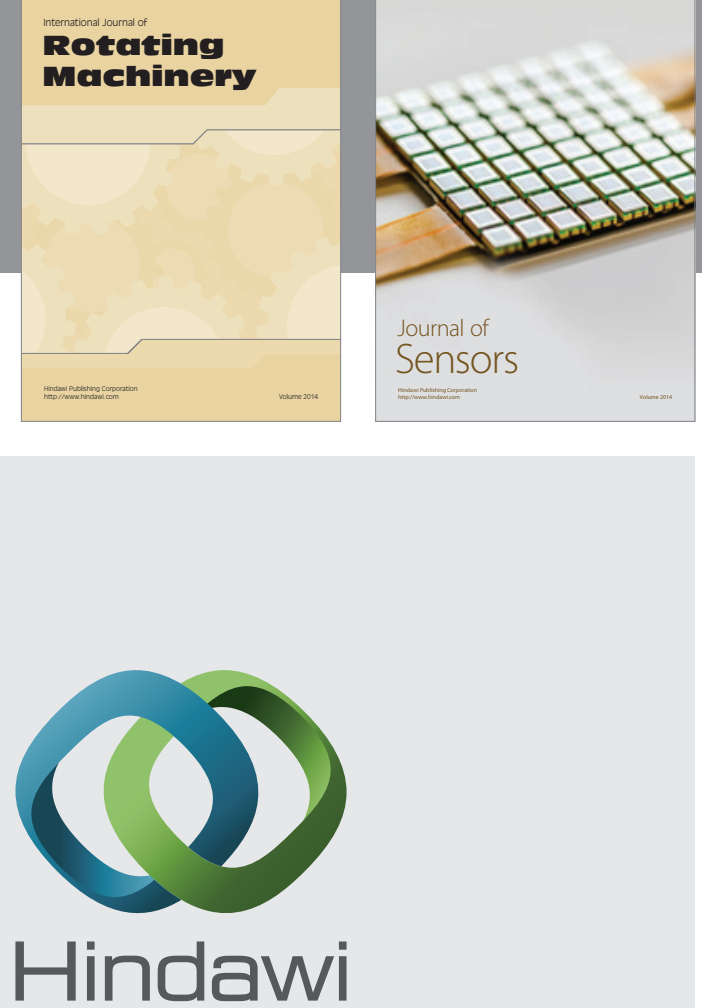

Submit your manuscripts at http://www.hindawi.com
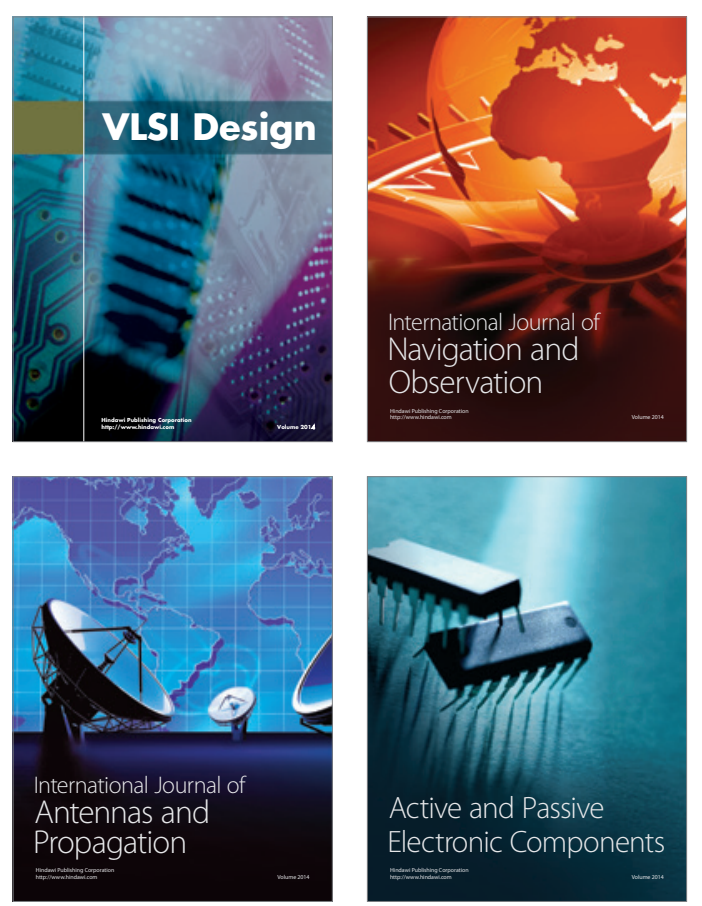
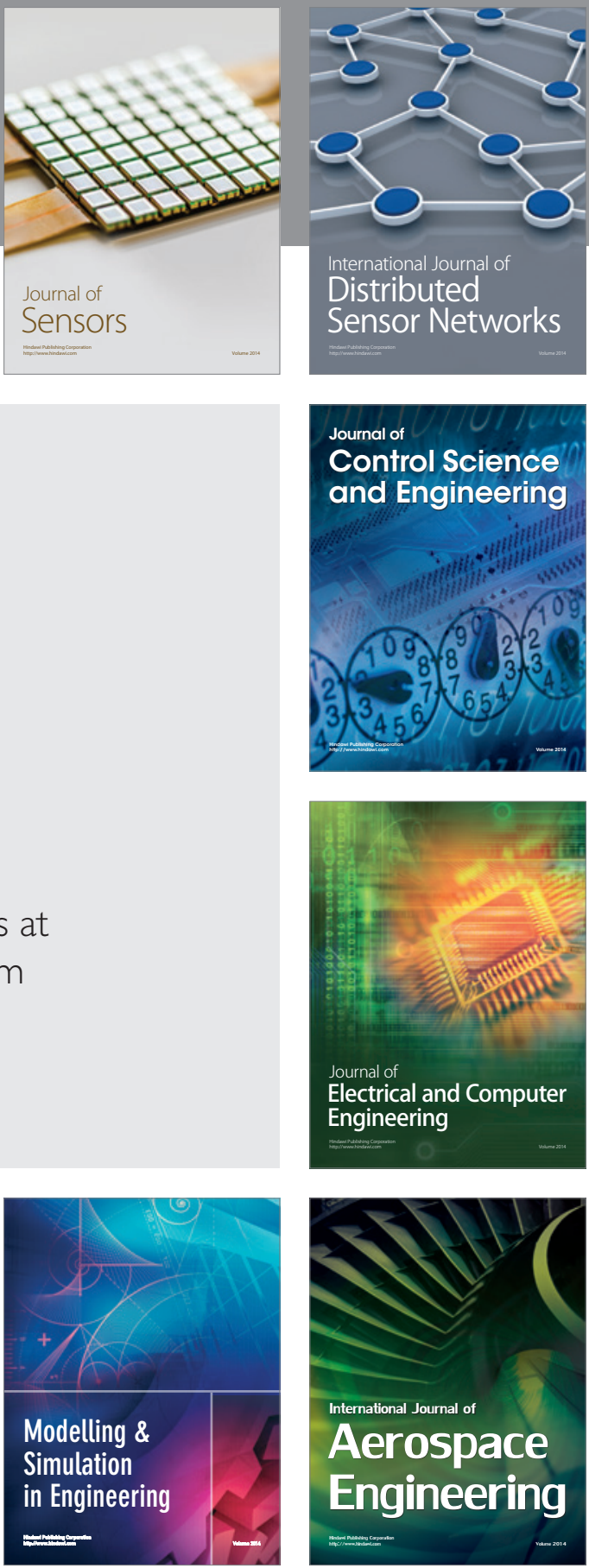

Journal of

Control Science

and Engineering
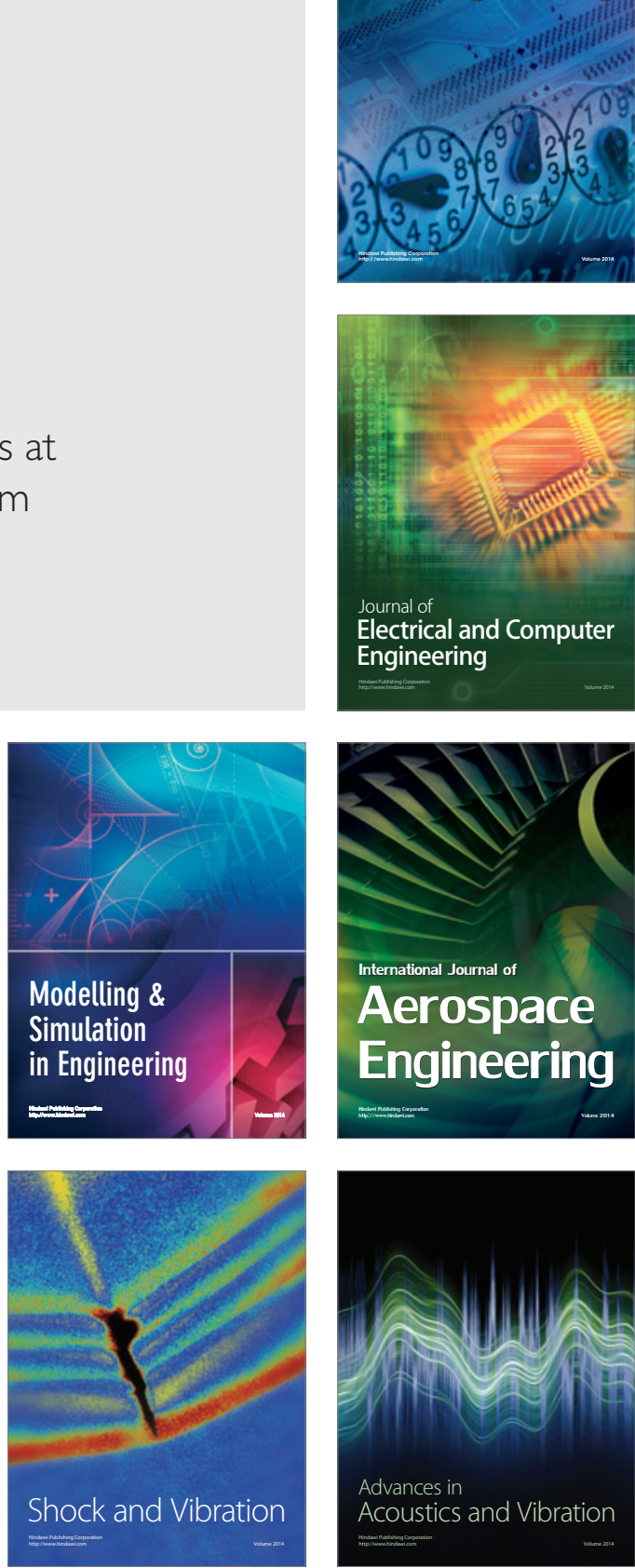